\title{
Die Formierung der anglo-schottisch-irischen Union im 18. Jahrhundert
}

\author{
JÜRGEN ELVERT
}

Mit seinen 1689/90 erschienenem Werk "Two Treatises on government" hatte sich der Philosoph, Erzieher und Arzt John Locke im ausgehenden 17. Jahrhundert Einlass in den Kreis der führenden staats- und verfassungsrechtlichen Theoretiker seiner Zeit verschafft. Locke war im Gefolge Wilhelm von Oraniens nach jahrelangem holländischem Exil wieder in seine Heimat zurückgekehrt. In den Niederlanden war er über die philosophischen Schriften Spinozas und die natur- und verfassungsrechtlichen Überlegungen Grotius' mit dem Gedankengut der holländischen Aufklärung in Berührung gekommen, die sein weiteres Leben und Wirken nachhaltig beeinflussten und ihn zu einem der Väter des englischen enlightenment werden ließen. Bereits vor seinem Zwangsaufenthalt auf dem Kontinent hatte er sich als ein finanziell unabhängiger, nicht korrumpierbarer und politisch unbequemer Denker erwiesen, für den das göttliche Recht königlicher Herrschaft keine Selbstverständlichkeit war, der sich aber auch entschieden gegen den von Thomas Hobbes in seinem „Leviathan“ (1651) eher weltlich definierten Staatsabsolutismus aussprach. Insgesamt bot er in seinem Werk einen auf die damals aktuellen Verhältnisse in England zugeschnittenen Verfassungsentwurf, als dessen wichtigstes Kriterium die Teilung der Macht in zwei Gewalten gilt, in die Legislative des Parlaments und die dem Parlament unterstellte Exekutive der Krone. ${ }^{1}$

Mit den „Treatises“ erschienen in England noch weitere sowie insgesamt 19 Neuauflagen älterer staatsrechtlicher Schriften. ${ }^{2}$ Dies sind Indizien dafür, wie sehr in der Blütephase der Aufklärung die Frage nach neuen Rahmenbedingungen staatlicher Herrschaft und Verfassung die Zeitgenossen bewegte. Mit der glorious revolution war die entscheidende staatsrechtliche Weichenstellung vorgenommen worden, durch die

1 Zu Locke vgl. z. B. Richard Ashcraft, Revolutionary Politics \& Locke's Two Treatises of Government, Princeton 1986.

2 Vgl. Conal Condren, George Lawson's Politica and the English revolution, Cambridge 1989, S. 151. 
das ehemals göttliche Recht der Krone der Souveränität des Parlaments unterworfen wurde, das fortan - wenigstens theoretisch - die Krone samt ihrer Rechte und Pflichten wie ein gewöhnliches Staatsamt vergeben konnte. Zwar blieben in der Regierungspraxis der kommenden Jahrzehnten bedeutende königliche Prärogativrechte wie die Wahl der Minister und die Zielvorgaben der, Großen Politik', besonders der Außenpolitik, weiterhin erhalten, doch bildete sich im Verlauf des 18. Jahrhunderts ein System der Checks and Balances zwischen der Krone, dem Oberhaus und dem Unterhaus heraus, das die staatliche Herrschaft beim ,König im Parlament' sah.

In Irland erwartete die protestantische Oberschicht vom Souverän dieselben Rechte und Machtbefugnisse, die die Bill of Rights der englischen nobility und gentry zugestand. Bei Anerkennung der Krone als Ausdruck anglo-irischer Gemeinsamkeit ging man dort von einer Neuverteilung der Kompetenzen nach englischem Vorbild zu Gunsten des Parlaments und zu Lasten des Vizekönigs oder seines Statthalters aus. Allerdings waren die der ,glorreichen Revolution' folgenden zwei Jahrzehnte in Irland von der Suche nach einer Neudefinition des anglo-irischen Verhältnisses geprägt. Dabei kristallisierten sich zwei grundsätzlich unterschiedliche Standpunkte heraus. Der eine, den J.G. Simms als „kolonialen Nationalismus “ bezeichnete, ${ }^{3}$ sah Irland als ein unabhängiges Königreich mit einem eigenen Parlament und eigenen funktionsfähigen staatlichen Organen. Dem englischen Parlament wurden nur wenige, eng begrenzte Mitspracherechte zugestanden. Einer der führenden irischen Denker der Zeit, William Molyneux ${ }^{4}$, fasste in seiner im Jahre 1698 erschienenen Schrift „The case of Ireland's being bound by acts of parliament in England, stated" die Argumente dieser Fraktion zusammen. Dabei leitete er den Unabhängigkeitsanspruch für Irland bereits aus den Verhandlungen ab, die seinerzeit zwischen den irischen Königen und Heinrich II. geführt worden waren. Einige allgemeinere Aussagen der Streitschrift, die sich mit der grundsätzlichen Gleichberechtigung der Menschen im Verhältnis zueinander und der Frage nach der freiwilligen Unterwerfung der Menschen unter Rechtsnormen als Charakteristikum einer zivilisierten Gesellschaft befassen, zeigen den großen Einfluss, den die Schriften John Lockes auch in Irland ausübten. ${ }^{5}$ Molyneux zählte zu einer relativ kleinen, aber an der Wende vom 17. zum 18. Jahrhundert gesellschaftlich einflussreichen Gruppe von Intellektuellen, der Dublin Philosophical Society ${ }^{6}$, zu deren Mitgliedern neben anderen politische Denker und Philosophen wie Robert Molesworth, John Toland und Jonathan Swift gehörten, der aber auch der

3 J.G. Simms, The establishment of protestant ascendancy, 1691-1714, in: T.W. Moody/W.E. Vaughan (Hg.), A New History of Ireland, Bd. 4: Eighteenth-Century Ireland 1691-1800, Oxford 1986, S. 5.

4 Zu Molyneux vgl. z. B.: J. G. Simms, William Molyneux of Dublin. A Life of the seventeenth-century Political Writer and Scientist, Dublin 1982.

5 Molyneux zum Beispiel führte eine intensive Korrespondenz mit Locke.

6 K. Theodore Hoppen, The Common Scientist of the Seventeenth Century. A Study of the Dublin Philosophical Society, Bd. 15: 1683-1708, London 2013. 
junge George Berkeley nahestand. In England stieß diese Interpretation auf heftigen Widerspruch. Eine Reihe von mehr oder weniger polemischen Gegendarstellungen versuchte, die Unhaltbarkeit der Argumente von Molyneux zu beweisen, auch sah sich das Unterhaus zu einer Resolution genötigt, die eine Bestandsgefährdung des Königreiches für den Fall vorhersagte, dass sich solche und ähnliche Überlegungen jenseits der Irischen See durchsetzten. Für eine Mehrheit im House of Commons bestand kein Zweifel daran, dass Irland ein von England abhängiges und untergeordnetes Königreich war. Mit dieser Einschätzung wussten sich die Abgeordneten in Übereinstimmung mit dem anderen in Irland vertretenen Standpunkt. Dieser sah das Land als ein von der Krone erobertes Territorium, das von der protestantischen Macht, die als eine Art zivilisatorischer Brückenkopf diente, für die Krone gehalten würde. Im Rahmen solcher Überlegungen gab es selbstverständlich auch kein gleichberechtigtes irisches Parlament, es musste stattdessen der englischen Herrschaft untergeordnet sein. Die Unvereinbarkeit der beiden Interpretationen verhinderte jahrelang eine sinnvolle $\mathrm{Zu}$ sammenarbeit zwischen den Vizekönigen und ihren irischen Statthaltern, die kraft Amtes den englischen Standpunkt zu vertreten hatten, und dem Parlament in Dublin, das mehrheitlich die Molyneux'sche Sichtweise unterstützte.

Stattdessen erzeugten ständige Dispute zwischen Legislative und Exekutive beiderseits der Irischen See Reibungsverluste, die die Klärung manches wichtigen politischen Problems über Gebühr verzögerte oder gar gänzlich verhinderte. Wie problematisch die ungeklärten Beziehungen sich auch auf das innerenglische Verhältnis zwischen König und Parlament auswirken konnten, zeigt die Kritik Westminsters an der Entscheidung Wilhelms, nach dem Frieden von Rijswijk (1697) gegen den erklärten Willen der Legislative nicht alle seine Söldnerheere aufzulösen, sondern Teile nach Irland zu verlegen. Das Parlament sah darin einen Versuch des Königs, sich außerhalb des unmittelbaren parlamentarischen Zuständigkeitsbereichs eine nur schwer kontrollierbare Machtbasis aufbauen zu wollen.

Für das irische Parlament bedeutete das eine Niederlage. Unmittelbar nach dem Sieg Wilhelms über Jakob hatten einflussreiche irische Protestanten sich ein ehrgeiziges Ziel gesteckt: die Vollendung der Union des irischen mit dem englischen Königreich. Zunächst waren dies Einzelmeinungen gewesen, doch vor dem Hintergrund der Diskussion um die konstitutionelle Stellung Irlands im Herrschaftsbereich der englischen Krone wurden nach dem Tod Wilhelms solche Forderungen immer öfter vorgetragen, im Jahre 1703 hatte das irische Unterhaus dann förmlich an Königin Anna appelliert, eine Union der beiden Königreiche herbeizuführen. Die Unionsdebatte wurde in enger Verbindung mit der Frage nach weitergehenden Rechten für das irische Parlament geführt. Schon Molyneux hatte in seiner Schrift dieses Junktim hergestellt, als er darauf hinwies, dass dem englische Parlament nur dann ein Mitspracherecht in irischen Angelegenheiten eingeräumt werden dürfe, wenn irische Abgeordnete in Westminster mit in den Abstimmungsprozess einbezogen würden. 
Und in der Folgezeit hatten irische Parlamentarier ebenso wie politische Theoretiker gern betont, dass eine Union mit England stets nur die zweitbeste Lösung nach einer konstitutionellen Absicherung der eigenen Entscheidungsfreiheit unabhängig vom englischen Parlament sein könne.

Trotz der unbestreitbaren Vorteile, die eine Vereinigung der beiden Königreiche den Angehörigen der protestantischen Elite Irlands, der sog. Protestant Ascendancy bot - es hätte die protestantische Minderheit des Landes aus der relativen sozialen Isolation im Lande befreit und die von ihr dominierte irische Wirtschaft enger an das englische ökonomische System gebunden - bleibt festzuhalten, dass sich in der protestantischen Oberschicht Irlands zu Beginn des 18. Jahrhunderts eine eigenständige kolonial-nationale Identität herauszubilden begann. Diese Entwicklung blieb nicht auf Irland beschränkt, sondern beeinflusste in den Folgejahren die politischen Entwicklungen auch außerhalb der engen Grenzen der Britischen Inseln. Vergleichbare Überlegungen jenseits des Atlantiks gipfelten im amerikanischen Unabhängigkeitskampf. Nachweislich waren die Schriften von Molyneux, Molesworth, Toland und einiger anderer verwandter politischer Denker im Verlauf des 18. Jahrhunderts in den nordamerikanischen Kolonien mit großem Interesse rezipiert worden. So konnten die Gedanken Lockes den amerikanischen Unabhängigkeitskampf auf zweifache Weise beeinflussen: Einmal indirekt über die irisch-protestantischen Staatstheoretiker, zum anderen direkt über die Rezeption durch die Vordenker der Revolution, die sich selbst oft und gerne auf Locke beriefen.

Es verwundert daher nicht, wenn die Nachricht vom Zusammenschluss Englands und Schottlands zum United Kingdom of Great Britain unter Auslassung des Königreichs Irland im Jahre 1707 die politischen Entscheidungsträger in Dublin nicht allzu sehr betrübte. Zwar verfasste Swift unter dem Eindruck der soeben vollzogenen anglo-schottischen Union die „Story of the injured lady“, in der Irland als ein junges Mädchen erscheint, das von einem Verehrer schmählich zu Gunsten einer hässlichen Rivalin sitzen gelassen wird - doch verzichtete er später auf eine Veröffentlichung dieser Allegorie. Und auch wenn sich das irische Parlament noch nicht ganz damit abfinden wollte - zwei Jahre später suchte das Oberhaus noch einmal um Vollzug der Union mit Großbritannien nach - scheint beiderseits der Irischen See das tatsächliche Interesse am Zusammenschluss der Königreiche nicht allzu groß gewesen zu sein. ${ }^{7}$

Trotz der Distanz, auf die das irische Parlament in der Frage des Verhältnisses zu Großbritannien gegangen war, wirkten sich die dortigen politischen Entwicklungen auch weiterhin unmittelbar auf Irland aus. Hier wie dort gab es Whigs und Tories, wenngleich in Dublin die Trennungslinien zwischen den politischen Lagern aufgrund der mehrheitlichen Sympathien für den kompromisslos anti-katholischen Kurs der

7 Bei Simms, 1986 (wie Anm. 3), S. 2ff., werden einige Hinweise darauf gegeben, die sich zum Beispiel auf bewussten Verzicht der irischen Exekutive in Bezug auf die Einbringung von Haushaltsentwürfen beziehen, die fortan vom Unterhaus vorgelegt wurden. 
britischen Whigs anders verliefen als in London. So standen die irischen Whigs dem anglikanischen Protestantismus der königstreuen britischen Tories beispielsweise näher als dem teils fundamentalistisch-sektiererischen Protestantismus vieler britischer Whigs. Die besonderen Rahmenbedingungen der protestant ascendancy Irlands, die sich ihrer Rolle als Minderheit in einer grundsätzlich immer noch feindlich eingestellten und mehrheitlich katholischen Gesellschaft durchaus bewusst war, bedingten folglich ungewöhnliche politische Konstellationen, die wiederum die Differenzen zur britischen Politik deutlich hervortreten ließen.

In den 1720er Jahren war der koloniale Nationalismus der protestant ascendancy ausgereift genug, um vor einer Auseinandersetzung mit der Krone nicht mehr zurückzuschrecken, wenngleich diese auch stellvertretend mit dem britischen Kronrat und dem Vizekönig geführt wurde. Schon bald entwickelte sich der Disput zu einer Grundsatzdebatte um die Entscheidungsgewalt des irischen Parlaments. Einer der Wortführer in der Debatte auf irischer Seite war Jonathan Swift, der sich in vier seiner „Drapier's letters“, publiziert zwischen Februar und Oktober 1724, jede britische Einflussnahme in irische Angelegenheiten verbat und statt dessen, wenn auch noch verschleiert, die völlige Unabhängigkeit des Königreichs Irland einforderte. ${ }^{8}$ Nur dank einer geschickten Regie der britischen Regierung in Verbindung mit dem irischen Vizekönig und dem irischen Parlaments gelang es, einen Ausweg aus der Verfassungsdebatte zu finden: Die protestant ascendancy akzeptierte die Obergewalt des britischen Parlaments, dieses verzichtete im Gegenzug für über zwanzig Jahre nahezu völlig darauf, seine Hoheitsrechte auch tatsächlich auszuüben.

Ohnehin waren die Jahre zwischen 1725 und 1750 durch ungewöhnliche politische Ruhe und Stabilität überall auf den britischen Inseln gekennzeichnet. In Irland hatten sich die protestantischen Grundherren spätestens nach dem Ausgleich mit London endgültig als führende gesellschaftliche Gruppe etablieren können. Aus einer derart saturierten Position heraus konnten sie es sich nun auch leisten, auf eine allzu harte Verfolgung von Andersdenkenden wie Katholiken oder Presbyterianern zu verzichten. Das war in den ersten Jahrzehnten des Jahrhunderts durchaus nicht selbstverständlich gewesen. Nach 1691 hatte in Irland eine kleine Minderheit protestantischer Landbesitzer einer katholischen Mehrheit gegenübergestanden. Exakte Zahlenangaben über das proportionale Verhältnis gibt es nicht, doch haben Berechnungen für die 30er Jahre des Jahrhunderts eine geschätzte Einwohnerzahl von zirka 3,1 Millionen mit einem katholischen Anteil von etwa 70\% ergeben. Damit hatte sich das absolute Verhältnis zwischen Katholiken und Protestanten seit den Tagen der glorious revolution kaum verändert, doch während sich 1688 noch etwa $22 \%$ des Landes in katholischer Hand befunden hatten, waren dies im Jahre 1703 nur noch

8 Vgl. Oliver W. Ferguson, Jonathan Swift and Ireland, Champaign 1962. 
14\%, bis 1776 ging dieser Anteil auf unter 5\% zurück, die restlichen 95\% gehörten ungefähr 5.000 protestantischen Grundbesitzern.

Die 50er Jahre des 18. Jahrhunderts sind in Bezug auf das Verhältnis der irischen Katholiken zur protestantischen Herrschaft von einem grundlegenden Stimmungsumschwung gekennzeichnet. Viele irische Katholiken, besonders Angehörige der katholischen gentry und der Kaufmannschaft, begannen, sich im Zeitalter der langsam entstehenden Geschichtswissenschaft mit der Suche nach der eigenen Identität zu beschäftigen. Dieses neu erwachte Interesse an der eigenen Vergangenheit lässt sich zwar durchaus mit geistesgeschichtlichen Entwicklungen anderswo in Europa vergleichen, doch besaß die irische Variante eine Besonderheit. Die Erinnerung an die eigene keltisch-irische Kultur konnte über eine Befriedigung rein historischer Neugierde hinaus trefflich als Beweis für die Daseinsberechtigung eigener keltisch-irisch-katholischer Werte innerhalb des protestantisch dominierten Irland dienen. Ohne es wissen zu können, eröffneten sie damit eine Debatte, die einige Jahrzehnte später den irischen Nationalismus des 19. und frühen 20. Jahrhunderts maßgeblich mit beeinflussen sollte.

Die offensichtliche Suche vieler irischer Katholiken nach einem Arrangement mit der protestant ascendancy setzte nicht unbedingt die Akzeptanz des politischen Status quo voraus. Dazu waren die Lebensbedingungen unter den Zwängen der penal laws entschieden zu schlecht. Nach wie vor jedoch hofften sie, sich des königlichen Wohlwollens durch die demonstrative Loyalität zur Krone zu versichern und dadurch möglicherweise einen größeren Handlungsspielraum gegenüber den irischen Protestanten zu gewinnen. So boten sie im Jahre 1762, als sich Großbritannien im Siebenjährigen Krieg einer französisch-österreichisch-spanischen Koalition gegenüberstand, der Londoner Regierung die Bildung eines irisch-katholischen Regiments an, das in der Armee des mit Großbritannien verbündeten Portugal kämpfen sollte. Doch die Dubliner Regierung protestierte energisch gegen die Bildung einer bewaffneten ,papistischen' Macht auf irischem Boden, und der Plan wurde rasch wieder fallengelassen.

Dennoch stand die Frage nach dem Verhältnis der einzelnen Reichsgebiete zueinander und zum Mutterland auf der politischen Tagesordnung in vielen Teilen des Empire. In den nordamerikanischen Kolonien hatte der britische Sieg über die Franzosen eine jahrelange Bedrohung beseitigt, die lange Zeit nur dank der militärischen Hilfe des Mutterlandes abgewehrt werden konnte. Nun war die Schutzmacht vor den imperialen Ambitionen Frankreichs überfüssig geworden, stattdessen gerieten die anglo-amerikanischen Beziehungen in eine tiefe Krise, ausgelöst durch zwei gegensätzliche Entwicklungen beiderseits des Atlantiks. In Nordamerika hatte sich wie in Irland ein koloniales Nationalbewusstsein entwickelt. Dieses bestimmte in zunehmendem Maße die Entscheidungen der politisch tragenden Schicht, also in erster Linie der Nachfahren der frühen Siedler, die sich in ihrer Argumentation und in 
der Definition ihrer politischen Ziele zumindest zu Beginn der Auseinandersetzung oftmals am irischen Modell orientierten. Ausgangspunkt des anglo-amerikanischen Disputs war die Unzufriedenheit in den Kolonien über die Form ihrer Repräsentation im Parlament von Westminster, wo nur eine kleine Gruppe nordamerikanischer Parlamentsabgeordneter vertreten war. Als in Großbritannien die Rechnungen aus dem Siebenjährigen Krieg fällig wurden, versuchte die Regierung zunächst, den zusätzlichen Finanzbedarf durch Steuer- und Abgabenerhöhungen zu decken. Die Vertreter und Sprecher der nordamerikanischen Kolonien indes verknüpften nach der Veröffentlichung dieser Absichten ihre Zustimmung dazu mit der Forderung nach verbesserter parlamentarischer Repräsentation - no taxation without representation wurde schnell zum Schlachtruf der frühen Unabhängigkeitskämpfer. Die Antwort aus London ließ nicht lange auf sich warten: In Erinnerung an die Auseinandersetzung mit den kolonialen Nationalisten der irischen protestant ascendancy erhielt das britische Parlaments per Gesetz die letztendliche Zuständigkeit für die nordamerikanischen Kolonien. Der Text dieses Gesetzes indes war in den entscheidenden Passagen identisch mit der „Declatory Act“ von 1719/20. Während das irische Parlament damals von vornherein lediglich versucht hatte, dessen Vorschriften durch Missachtung zu unterwandern und letztlich einen Kompromiss mit Westminster erreichen konnte, sollte sich diesmal daran ein Funke entzünden, der nur zehn Jahre später, am 4. Juli 1776, zur Unabhängigkeitserklärung der 13 ehemaligen nordamerikanischen Kolonien Großbritanniens führte.

Die wachsenden Spannungen jenseits des Atlantiks wurden in Irland aufmerksam verfolgt. Seit dem frühen 18. Jahrhundert war ein steter, wenn auch nicht allzu starker, Strom von Emigranten, hauptsächlich aus Ulster, in die nordamerikanischen Kolonien geflossen und hatte auf diese Weise persönliche transatlantische Bindungen entstehen lassen, die entscheidend dazu beitrugen, dass die nordamerikanischen Siedler von vielen Iren als Schicksalsgenossen betrachtet wurden - dies umso mehr, als ihnen die Motive der amerikanischen Unabhängigkeitsbewegung wohlbekannt waren. Seit Ausbruch der Unruhen in den Kolonien hatten daher fast alle gesellschaftlichen Gruppen des Landes Solidaritätsadressen an die Amerikaner verabschiedet. Zahlreiche Zeitungen forderten zudem von der Dubliner Legislative und Exekutive, auf König Georg III., seine Regierung und das Parlament von Westminster dahingehend einzuwirken, den Konflikt mit friedlichen Mittel beizulegen. Allerdings wurde ein aktives irisches Eingreifen zugunsten der rebellierenden Amerikaner kaum gefordert im Gegenteil: Als sich der Konflikt durch das Eingreifen von Frankreich und Spanien auszuweiten drohte, stellte sich die politische Führung Irlands fast geschlossen hinter die britische Politik.

Die Einflüsse des amerikanischen Unabhängigkeitskrieges auf die irische Wirtschaft, Gesellschaft und Politik waren beträchtlich. Das im Jahre 1776 verhängte Exportverbot für Nahrungsmittel ließ nur noch Ausfuhren nach Großbritannien und 
in die nicht an der Rebellion beteiligten Kolonien zu. Damit versuchte die britische Regierung, die zur Versorgung der Truppen in Nordamerika Nahrungsmittel in großem Umfang einkaufen musste, die Preise so stabil wie möglich zu halten. Viele Produzenten in Irland protestierten anfangs energisch gegen solche Eingriffe und bezeichneten sie als den verfassungswidrigen Versuch, unliebsame Konkurrenz auszuschalten. Doch waren ihre Sorgen nur teilweise begründet, denn trotz aller Anstrengungen der britischen Regierung ließ die große Nachfrage nach Nahrungsmitteln die Preise kontinuierlich ansteigen, so dass die Absatzeinbußen wieder annähernd ausgeglichen werden konnten. Weit nachhaltigere Auswirkungen hatten die Rufe vieler britischer Grundbesitzer, die protektionistische Maßnahmen gegen irische Produkte zum Schutze der eigenen Erzeugnisse forderten. Sie belebten in Irland die Unabhängigkeitsdebatte neu, die, auch vor dem Hintergrund der aktuellen Ereignisse in Nordamerika, für die nächsten Jahre wieder das grand thème der heimischen Politik sein sollte. Im irischen Parlament mehrten sich erneut die Stimmen, die unverblümt die völlige irische Unabhängigkeit forderten. Sie sammelten sich um eine ursprünglich eher lose Verbindung von protestantischen Abgeordneten kolonial-nationaler Provenienz, die sich selbst den Namen Patriots gegeben hatten. Die Argumente, die sie benutzen, waren durchaus fundiert, hatte doch die britische Regierung in den vergangenen Jahren mehrfach heimische Interessen irischen Wünschen vorgezogen, gleichzeitig jedoch keine Zweifel an der Entschlossenheit aufkommen lassen, die Fäden politischer Macht in Irland auch künftig fest in den eigenen Händen halten zu wollen.' Im Umkehrschluss folgerten nun viele Angehörige der protestant ascendancy, dass die irische Unabhängigkeit die beste Voraussetzung für eine wirklich gedeihliche Zusammenarbeit mit Großbritannien sein würde - diese wurde selbst nicht in Frage gestellt, sondern als Grundlage der eigenen politischen Macht vorbehaltlos anerkannt. Prominentester Wortführer dieser Gruppe war der 1775 zum Führer der Patriots gewählte Unterhausabgeordnete Henry Grattan ${ }^{10}$.

Der Kriegsverlauf lieferte den irischen ,Patrioten' zusätzliche Argumente und förderte nachhaltig die Verbreitung ihrer politischen Ideen. Seit Ausbruch der Unruhen in den amerikanischen Kolonien, also seit Beginn der 70er Jahre, waren ständig Truppen aus Irland abgezogen worden. Dies schien durchaus vertretbar gewesen zu sein, da sich die innenpolitischen Verhältnisse des Landes wesentlich stabilisiert hatten und auch die irischen Katholiken offensichtlich den Status quo akzeptierten. Doch der Kriegseintritt Frankreichs und Spaniens änderte die strategische Lage Irlands und Britanniens grundlegend. Beiderseits der Irischen See erwachten lang vergessene Invasionsängste neu. Um einen wirkungsvollen Schutz vor Überfällen von

9 Vgl. dazu die detaillierte Schilderung der politischen Entwicklungen in den 60er und 70er Jahren in: R. B. McDowell, Ireland in the Age of Imperialism and Revolution, 1760-1801, Oxford 1979, dort bes. S. 209-274.

10 Zu Grattan vgl. R. B. McDowell, Grattan. A life, Dublin 2001. 
außen zu haben und über einen ausreichend großen Sicherheitsapparat im Falle von inneren Unruhen verfügen zu können, wurden mindestens 12.000 Soldaten benötigt. Im Jahre 1778 jedoch standen nur noch etwa 8.000 Mann in Irland unter Waffen. Die Gefahr einer Landung französischer Truppen in Großbritannien oder Irland war durchaus gegeben, zumal sich die meisten Einheiten der britischen Flotte in Übersee befanden und Frankreich, wenigstens zeitweilig, die Seeherrschaft im Kanalgebiet und in der Irischen See wiedererlangt hatte. Auch operierten in diesen Jahren einige amerikanische Kaperschiffe in den Gewässern rund um Irland, die dort relativ ungestört und erfolgreich Jagd auf britische Handelsschiffe machten. Die protestantischen Grundherren reagierten diesmal ungewöhnlich schnell auf die Herausforderung. Da die Regierung in Dublin nicht mehr über genügend Truppen verfügte, um die Küstenverteidigung aus eigener Kraft sicherzustellen, entstanden innerhalb weniger Wochen überall im Lande größtenteils aus privater Initiative der jeweiligen regionalen Magnaten oder kleinerer Gruppen von Grundbesitzern bewaffnete Milizverbände. Bei den Angehörigen dieser Verbände handelte es sich überwiegend um Freiwillige aus den Reihen der Pächter und der Kaufmannschaft, sie nannten sich daher schlicht Volunteers.

Deren fast ausnahmslos protestantisches Offizierskorps entstammte zumeist der gentry, während Unteroffiziere und Mannschaften überwiegend Landpächter, Kaufleute und Handwerker, also allesamt Angehörige der damaligen Mittelklasse waren. Obwohl die katholische Bevölkerung mehrfach versucht hatte, ihre Loyalität zum Staat zu beweisen, schreckten die meisten protestantischen Grundherren noch immer vor einer Aufnahme von katholischen Freiwilligen zurück, abgesehen davon, dass sich viele Katholiken eine Mitgliedschaft bei den Volunteers gar nicht leisten konnten, da diese für ihre Ausrüstung und Verpflegung selbst aufkommen mussten. So blieben die Angehörigen der ascendancy überwiegend unter sich. Doch da in den Korps die sozial einflussreichsten Schichten vertreten waren, wurde die Mitgliedschaft in einem der Korps schnell zur gesellschaftlichen Ehrensache.

1782 zählten die verschiedenen Freiwilligenverbände landesweit bereits über 100.000 Mitglieder. Sie verkörperten die Enttäuschung weiter Teile der Bevölkerung über die politischen Verhältnisse der Zeit, besonders über die Abhängigkeit des irischen vom britischen Parlament. Die britische Regierung und der Vizekönig in Dublin hatten keine andere Wahl - sie mussten die neu entstandene außerparlamentarische Kraft in Irland zur Kenntnis nehmen, wollten sie nicht riskieren, dass die Spannungen kulminierten und die Unzufriedenheit der kolonialen Nationalisten Irlands in eine Unabhängigkeitserklärung nach amerikanischem Vorbild mündete. Angesichts des britischen Engagements jenseits des Atlantiks reichten die Heimattruppen bei weitem nicht aus, um der neuen Herausforderung erfolgreich mit militärischen Mitteln begegnen zu können. Die Regierung entschloss sich daraufhin, einigen zentralen Forderungen der irischen protestant ascendancy nach größerer poli- 
tischer und wirtschaftlicher Unabhängigkeit von London stattzugeben. ${ }^{11}$ Diese Maßnahmen wurden in Irland überwiegend wohlwollend, zum Teil sogar begeistert aufgenommen. Schließlich schienen auf den ersten Blick alle Wünsche nach politischer und wirtschaftlicher Unabhängigkeit in Erfüllung gegangen zu sein. Der Eindruck wurde durch die Einrichtung eines eigenen irischen Postwesens und einer irischen Nationalbank noch unterstrichen. Doch trotz der weitreichenden Zugeständnisse besaß London nach wie vor erhebliche, wenn auch überwiegend informelle, Mitspracherechte in irischen Fragen. Zwar war es nun das verbriefte Exklusivrecht des Parlaments in Dublin, Gesetze für Irland zu erlassen, doch Rechtswirksamkeit erhielten sie erst durch das Siegel des britischen Lordkanzlers, der wiederum vor der Besiegelung die Zustimmung des britischen Kabinetts einzuholen hatte. Ferner wurden der Vizekönig und sein Stellvertreter nach wie vor von der Krone ernannt und gehörten auch nach 1782 der jeweiligen britischen Regierung an. Trotz dieser offensichtlichen Defizite war die Mehrheit der irischen Parlamentarier indes mit dem Erreichten ebenfalls zufrieden, schließlich konnte man der britischen Krone nun Loyalitätsadressen zukommen lassen, ohne einen Gesichtsverlust im Lande fürchten zu müssen. Auch war es weiterhin möglich, an dem im Einzelfall oftmals durchaus profitablen Patronagesystem der Krone teilzuhaben.

Die Reformen sorgten dafür, dass die 1780er Jahre für die anglo-irischen Beziehungen ein relativ ruhiges Jahrzehnt sein sollten. In Irland wie in Großbritannien schien der Wunsch nach einer Atempause zur Erholung von den stürmischen Ereignissen, die den äußeren Rahmen ebenso wie die inneren Strukturen des Empire so nachhaltig verändert hatten, das politische Leitmotiv dieser Jahre zu sein. Dennoch blieben viele Fragen, besonders in Bezug auf die Gestaltung der wirtschaftlichen Zusammenarbeit, offen. Die Notwendigkeit einer Überarbeitung der handelspolitischen Beziehungen zwischen beiden Ländern waren schon in den frühen 80er Jahren festgestellt worden, als in Folge einer Missernte in Irland Stimmen laut wurden, die protektionistische Maßnahmen gegen britische Agrarerzeugnisse verlangten. Diese Reaktion forderten jene Kräfte in beiden Hauptstädten heraus, die sich der Schaffung föderaler Reichsstrukturen ohne Handelsschranken verschrieben hatten. Sie erkannten, dass ein solches Ziel nur durch ein Vertragswerk erreicht werden konnte, in das die gegenseitigen Wirtschaftsbeziehungen ebenso wie Sicherheitsfragen mit einbezogen sein würden. ${ }^{12}$

Der irische Hauptgesprächspartner bei den Entwurfsverhandlungen war Henry Grattan, zu dieser Zeit immer noch Sprecher der Patriots, mittlerweile jedoch die dominierende Persönlichkeit im irischen Unterhaus schlechthin. Es gelang ihm, den

11 Vgl. Tony Claydon / Ian McBride (Hg.), Protestantism and National Identity. Britain and Ireland, c. 1650c. 1850, Cambridge 1999.

12 Vgl. Liam de Paor, The Peoples of Ireland. From Prehistory to Modern Times, New York 1986, S. 206. 
Briten noch zwei weitere Konzessionen abzuringen: Bei Inkrafttreten des Vertrages wäre dem irischen Parlament ein unabhängiges Kontrollrecht der Beträge zum Ausbau der Royal Navy vorbehalten worden, womit es ein direktes Mitbestimmungsrecht in Verteidigungsangelegenheiten erhalten hätte. Ferner sollte das Abkommen zunächst das irische Parlament passieren, bevor es in Westminster zur Entscheidung vorgelegt worden wäre. Die erste Hürde bereitete keinerlei Schwierigkeiten, doch in Westminster verursachte der Entwurf eine wahre Welle der Entrüstung und Empörung. Besonders die britischen Händler und Manufakturbetreiber wandten sich energisch gegen die Zugeständnisse an die irische Konkurrenz. Dabei nutzten sie alle ihnen zur Verfügung stehenden Mittel und Möglichkeiten aus, einschließlich einer intensiven und kostenaufwendigen Pressekampagne. Nachdem die Parlamentarier zwanzig Änderungsvorschläge formuliert hatten, wurde der so überarbeitete Entwurf mit der denkbar knappen Mehrheit von 20 Stimmen angenommen, nur um diesmal am 15. August 1785 nach der Rückverweisung im irischen Unterhaus zu scheitern.

Inhalt und letztliches Scheitern des geplanten Abkommens spiegeln den Stellenwert wieder, der beiderseits der Irischen See dem Verbleib Irlands im britischen Empire beigemessen wurde, sie reflektieren aber auch die grundsätzlich unterschiedlichen Auffassungen von der Art des Verbleibs. Premierminister Pitt setzte zunächst mit guten Gründen auf freiwillige Kooperation. Nach dem Ausscheiden der ehemaligen nordamerikanischen Kolonien aus dem Reichsverbund stellte Irland aufgrund seiner unmittelbaren geographischen Nähe zum Mutterland des Empire den Prüfstein für die weitere Funktionsfähigkeit imperialer Zusammenarbeit und für die Durchsetzungskraft Großbritanniens im imperialen Verbund dar. Ein Ausscheiden des unmittelbaren Nachbarn hätte auf die überseeischen Territorien eine deutliche Signalwirkung ausgeübt und diese vielleicht dem nordamerikanischen und irischen Beispiel folgen lassen. Es wäre ferner von rivalisierenden europäischen Mächten mit imperialen Ambitionen wie zum Beispiel von Frankreich, Spanien oder den Niederlanden zu Recht als ein Zeichen der Schwäche interpretiert worden und hätte möglicherweise bewaffnete Konflikte in Übersee um dortige Besitzungen nach sich gezogen, denen London nach dem kräftezehrenden Kampf in Nordamerika noch nicht gewachsen war. Ein im beiderseitigen Einverständnis geschlossener Vertrag jedoch, der den freiwilligen Verzicht auf bestimmte Hoheitsrechte kodifiziert hätte, wäre international als Symbol für die Handlungsfähigkeit des British Empire gewertet worden. Aus reichs- und außenpolitischer Perspektive sind die weitgehenden Zugeständnisse, mit denen die britische Regierung die kolonialen Nationalisten in Irland zum Verbleib im Empire bewegen wollte, also durchaus nachvollziehbar. Der heftige Protest, auf den der Vertragsentwurf im Londoner Parlament stieß, war hauptsächlich Ausdruck der Angst vieler britischer Unternehmer und Kaufleute vor einem Verlust liebgewonnener Privilegien, die sie massiven Druck auf Westminster ausüben ließ. Dieser Druck war indes durchaus willkommen, hätte die Akzeptanz des Abkom- 
mens doch einen realen Machtverlust beider Häuser zur Folge gehabt. Darauf indes brauchten die Mitglieder des House of Commons bei den Nachbesserungen nun nicht mehr explizit hinzuweisen.

Angesichts des prononcierten Kolonialnationalismus in Irland barg die starre Haltung Westminsters das verhältnismäßig große Risiko in sich, eine Sezessionserklärung des irischen Parlaments zu provozieren, wo mittlerweile die meisten Abgeordneten den Patriots Henry Grattans zuzurechnen waren. Dieses Risiko war freilich kalkulierbar, hatte doch das Verhalten der irischen Parlamentarier in den zurückliegenden Jahren stets darauf schließen lassen, dass sie nicht daran dachten, den konstitutionell festgelegten Handlungsrahmen zu überschreiten. Zwar hatten Grattan und seine Patriots nach dem Scheitern des anglo-irischen Abkommens noch einmal nachdrücklich darauf hingewiesen, dass sie, vor die Wahl gestellt, zwischen Empire und Freiheit entscheiden zu müssen, stets für die Freiheit votieren würden, doch gelang es ihnen in den folgenden Jahren nicht, jene Integrationskraft zu entwickeln, die nötig gewesen wäre, um die gespaltene irische Gesellschaft hinter sich zu vereinen. Stattdessen erhielten am Rande der kolonialnationalen Bewegung solche Kräfte größeren Zulauf, die zu einem radikaleren Vorgehen auf dem Weg zur Unabhängigkeit von Großbritannien bereit waren. Doch für London schloss, schon die imperiale Raison eine irische Unabhängigkeit von vornherein aus. Nachdem die Vertragsinitiative gescheitert war, nahm Premierminister Pitt daher eine grundsätzliche Neuorientierung seiner Irlandpolitik vor, die allerdings mit den im irischen Parlament und in der irischen Gesellschaft entwickelten Vorstellungen über das bilaterale Verhältnis nicht mehr vereinbar war.

Die Wellen, die die revolutionären Entwicklungen in Frankreich schlagen ließ, trieben auch in Irland die Frage nach einer Neudefinition des irischen Selbstverständnisses schneller als bislang voran, möglicherweise dort aufgrund der jahrhundertealten guten familiären, religiösen, kulturellen und ökonomischen franko-irischen Beziehungen sogar noch schneller und radikaler als in anderen Teilen des Kontinents. Auch auf der irischen Insel lösten sie eine heftige Debatte über politische Grundsatzfragen aus, in denen die liberalen Kräfte nach Analogien zwischen den französischen Ereignissen und der glorious revolution suchten, während die Konservativen eher vor den Gefahren eines völligen Zusammenbruchs staatlicher Autorität warnten.

Vor diesem Hintergrund verbesserten sich die Beziehungen zwischen den Patriots im Dubliner Parlament und den Whigs im britischen House of Commons signifikant. ${ }^{13}$ Daraus entstand in der Folgezeit eine Art Kooperation, die bis zur Übernahme bestimmter, schon seit längerem von der britischen Opposition gestellter Forderungen durch Grattan und seine Mitstreiter führte. Diese bezogen sich zumeist auf Kritik

13 Vgl. dazu die entsprechenden Ausführungen bei: Clemens Körte, Rechtsbewusstsein und Verrechtlichung in der irischen Agrargesellschaft 1760-1850, Göttingen 2006. 
an der bestehenden Form parlamentarischer Repräsentation, an der Form der Patronage, der Ämtervergabe und anderer Mittel, die die Regierungspartei anwandte, um die Sitzmehrheit zu erhalten. Damit jedoch wurden auch die Bindungen der Patriots an das britische politische System wieder gestärkt, so dass sich Grattan im Sommer 1790, als die Spannungen zwischen Großbritannien und Spanien aufgrund rivalisierender Gebietsansprüche an der nordamerikanischen Westküste sich bedrohlich verstärkten und ein neuer Krieg nicht mehr ausgeschlossen werden konnte, zu einer öffentlichen Erklärung entschloss, in der er betonte, dass die Interessen Großbritanniens und Irland nicht voneinander zu trennen seien.

Dies stieß bei vielen engagierten irischen Kolonialnationalisten auf heftige Kritik. So veröffentlichte beispielsweise Theobald Wolfe Tone, ${ }^{14}$ ein junger protestantischer Dubliner Rechtsanwalt, daraufhin eine Flugschrift, in der er darauf hinwies, dass sich Irland nicht in einem Konflikt mit Spanien befinden würde und dass der Hinweis auf die Notwendigkeit irischer Hilfe für Großbritannien zum Wohle des Empire, den Grattan in seiner Loyalitätsadresse gegeben hatte, eine trügerische und hohle Phrase gewesen sei. In seiner berühmt gewordenen Schrift mit dem Titel „An argument on behalf of the catholics in Ireland" folgerte er im darauffolgenden Jahr, dass die einzige Möglichkeit, dem britischen Einfluss auf irische Angelegenheiten begegnen zu können, eine Parlamentsreform sei, die nur durch eine enge Zusammenarbeit der irischen Katholiken und der radikalen Protestanten erreicht werden könne. Tones Forderungen entsprachen den Ansichten vieler aufgeklärter Liberaler und radikaler Protestanten in Irland, sie wurden gleichfalls von den Führern der organisierten Katholiken nachdrücklich begrüßt. Überall im Lande trafen sich unter maßgeblicher Beteiligung der verschiedenen lokalen und regionalen Volunteer-Korps aufgeklärte Protestanten und Katholiken, um nach Wegen zu suchen, auf denen die geteilte irische Gesellschaft unter Berücksichtigung der Ideale der - zwischenzeitlich offensichtlich nicht mehr kontrollierbaren - französischen Revolutionäre zu einer, freien, gleichen und gerechten Gemeinschaft geführt werden sollte.

Besonders beliebt für solche Treffen waren die Jahrestage des Sturms auf die Bastille, und so wurden dann auch erstmals am 14. Juli $Z$ auf einer Bürgerversammlung in Belfast Überlegungen die Gründung einer politischen Vereinigung betreffend angestellt, in der gleichermaßen Protestanten wie Katholiken vertreten sein sollten. Die Ulster-Metropole, zu dieser Zeit ein etwa 20.000 Einwohner zählender aufstrebender Industriestandort, bot sich für ein solches Treffen besonders an, da im irischen Nordosten im Gegensatz zum eher staatskirchlich-konservativ orientierten Dubliner Verwaltungssitz aufgeklärtes Unternehmertum presbyterianischer Provenienz dominierte, das sich von der Bildung einer neuen Gesellschaft auf der Grundlage gemein-

$14 \mathrm{Zu}$ Tones Bedeutung bei der Formierung der United Irishmen vgl. Sean Cronin, Irish Nationalism. A History of Its Roots and Ideology, Dublin 1980. 
samer katholischer und presbyterianisch-protestantischer Interessen die Ablösung der alten Herrschaftsstrukturen erhoffte. Nur wenige Wochen später, am 14. Oktober 1791, wurde das Statut der Belfast Society of United Irishmen verabschiedet, an dessen Formulierung Theobald Wolfe Tone maßgeblich beteiligt war. ${ }^{15}$

Der starke Zulauf, den die United Irishmen mit ihrem konfessionsübergreifenden Ansatz verbuchen konnten, erweckte manche Organisationen zu neuem Leben, die längere Zeit kaum an die Öffentlichkeit getreten waren, sich nun aber mit ähnlich lautenden Forderungen wieder an die Öffentlichkeit wandten. Nach langen Jahren relativer Stabilität hatte der Reformprozess somit in kurzer Zeit eine brisante Gemengelage aus divergierenden politischen und sozialen Interessen entstehen lassen. Zwar verfügten die liberalen Abgeordneten um Henry Grattan nach wie vor über großen Einfluss im Parlament, doch ihre Bereitschaft zu weiteren Konzessionen an die Katholiken und zu einer moderaten Parlamentsreform, mit denen sie einen gesamtgesellschaftlichen Konsens erreichen wollten, stieß auf wachsende Kritik beiderseits der Irischen See. In London weckten überdies seit Anfang 1794 vertrauliche Hinweise auf Kontakte zwischen den United Irishmen und Repräsentanten des revolutionären Frankreichs alte Invasionsängste zu neuem Leben.

Diese Ängste und Sorgen waren nicht unbegründet. Aus Enttäuschung über den ihrer Meinung nach allzu langsamen Fortschritt des Reformprozesses hatten in der Tat einige führende Vertreter der United Irishmen, unter ihnen Tone, im April 1794 Verbindung mit dem französischen Nationalkonvent aufgenommen und diesem eine Liste von Bedingungen übergeben, unter denen sie eine Landung französischer Truppen in Irland unterstützen würden. Die Regierung war durch eigene Agenten über jede Phase dieser Konspiration informiert, konnte Jackson daher vor seiner Rückkehr nach Frankreich verhaften lassen und damit auch die Überbringung der Bedingungen verhindern, doch besaß sie außer dem begründeten Verdacht keinerlei Beweise, die gegen die irischen Verschwörer verwendet werden konnten. Diese nutzten ihre guten Kontakte zur vizeköniglichen Administration in Dublin, um einen allseits akzeptablen Ausweg aus dem Dilemma zu finden. Der Kompromiss sah vor, dass Tone alle Verantwortung an dem Treffen auf sich nahm, dafür nach Nordamerika ins Exil gehen durfte und die Regierung als Gegenleistung auf eine weitere Verfolgung von Angehörigen der - durch den Verlust eines fähigen Organisators und ausgewiesenen politischen Publizisten nun erheblich geschwächten - United Irishmen in dieser Angelegenheit verzichtete. ${ }^{16}$

In Ulster, besonders in der Grafschaft Armagh, schlossen sich zur selben Zeit Protestanten zu bewaffneten Geheimbünden, den sogenannten fleets zusammen. ${ }^{17}$

15 Vgl. Jürgen Elvert, Geschichte Irlands, ${ }^{4} 2003$, Kapitel 5.

16 Vgl. Tom Dunne, Rebellions. Memoir, Memory and 1798, Dublin 2004.

17 Zur Formierung von Geheimgesellschaften im revolutionären Irland vgl. Jürgen Elvert, Geheimbünde und 
Damit verstießen sie zwar gegen geltendes Recht, doch sah sich die ohnehin krisengeschüttelte Dubliner Regierung nicht in der Lage, dagegen einzuschreiten. Die Mitglieder einer dieser Verbände nannten sich selbst in Erinnerung an Wilhelm von Oranien Orange Boys. Aus dieser Gruppe sollte sich wenig später der Loyal Orange Order, die bis heute wirksamste außerparlamentarische anti-katholische pressure-group Ulsters herausbilden. ${ }^{18}$ Die Protestanten beriefen sich bei ihren Aktionen, die vornehmlich gegen katholische Pächter oder Weber gerichtet waren, auf das immer noch geltende Verbot des Waffenbesitzes für Katholiken. Dieses Gesetz würde, so hieß es, durch die Zugehörigkeit zu den Milizverbänden systematisch unterlaufen und sei eine permanente Bedrohung der eigenen Sicherheit. Die Reaktion der Katholiken ließ nicht lange auf sich warten. In kurzer Zeit entstanden landesweit eigene Geheimbünde, die sogenannten Defenders, die durch ein wirksames Netzwerk von Verbindungen eng miteinander kooperierten und sich nötigenfalls gegenseitig schnelle Hilfe zukommen lassen konnten. Im Gegensatz zu den protestantischen Gruppen besaßen die katholischen Geheimbünde sogar so etwas wie eine politische Programmatik. Diese jedoch war wenig differenziert und offensichtlich populistisch - man forderte niedrigere Pachtsätze, höhere Löhne für Landarbeiter sowie die Reduktion oder gänzliche Abschaffung des Zehnten. In ihren oftmals brutalen Methoden unterschieden sie sich indes nicht von ihren protestantischen Widersachern: anonyme Drohbriefe, öffentliche anonyme Verunglimpfung missliebiger Personen, Angriffe auf Haus und Hof der Gegner sowie Überfälle zur Beschaffung von Waffen.

Zunächst hielt sich die sektiererische Gewalt in Grenzen und war oftmals eher das Ergebnis zufälliger Aufeinandertreffen, während sich der alltägliche protestantisch-katholische Antagonismus in Ulster hauptsächlich im Wettstreit um gutes Ackerland, auf Viehmärkten oder auch, dann eher sportlich, bei Hahnenkämpfen zeigte. Das Jahr 1795 markiert indes einen Wendepunkt, denn von nun an wurde die Auseinandersetzung gezielter, planmäßiger und mit weitreichenderen Konsequenzen ausgetragen. So gelang es einer gut organisierten Gruppe protestantischer Peep-o'Day Boys im Jahre 1795 binnen weniger Wochen, in Nordarmagh etwa tausend Katholiken aus ihren Häusern zu vertreiben und zur Auswanderung in weniger dicht besiedelte Regionen, hauptsächlich nach Connacht, zu zwingen, ohne dass die Magistrate der betroffenen Gemeinden dagegen eingeschritten wären oder die Dubliner Regierung Truppen zum Schutz der nun existentiell bedrohten Katholiken in Marsch gesetzt hätten. Die Reaktion der Defenders ließ nicht lange auf sich warten. Im September terrorisierte eine Gruppe von ihnen wochenlang protestantische Farmen im Norden der Grafschaft. Es kam, wie es kommen musste: Die bedrohten Farmer riefen

kolonialer Nationalismus in Irland von der Mitte des 18. Jahrhunderts bis zum Vollzug der anglo-irischen Union von 1801, in: Volkhard Huth (Hg.), Geheime Eliten?, Frankfurt/ Main 2014, S. 345-362.

18 Vgl. Sean J. Connolly, Divided kingdom. Ireland, 1630-1800, Oxford 2008, S. 432. 
die Peep-o'-Day Boys um Hilfe, diese kamen nur zu gerne und stellten die katholische Sektierer in der Nähe von Loughgall an einem Ort namens The Diamond. ${ }^{19}$ Die besser ausgebildeten und ausgerüsteten Protestanten verließen das Schlachtfeld als Sieger und ließen über 30 tote Defenders zurück. In den folgenden Wochen und Monaten verstärkten sich die Übergriffe protestantischer Gangs auf unbeteiligte katholische Bewohner der Grafschaft, denen nun keine, Verteidiger' mehr zur Hilfe kommen konnten. Erneut sahen die Regierungsvertreter sowie ortsansässige landlords mehr oder weniger tatenlos zu. Nach wie vor verfügten sie selbst nicht über genügend Mittel, um diese Übergriffe zu verhindern, doch nicht ohne Grund befürchteten viele von ihnen, sollten sie trotzdem gegen einzelne fleets vorgehen, einen Übergriff des protestantischen Protestes auf andere Grafschaften Ulsters.

Zwischenzeitlich hatten sich sowohl die politischen Ziele als auch die organisationalen Strukturen der United Irishmen erheblich verändert. Sie mussten erkennen, dass weder die Regierung in Dublin, noch Regierung, Krone oder Parlament in London bereit waren, auf ihre umfassenden Reformpläne einzugehen. Stattdessen strebten sie nun die Errichtung einer Irischen Republik an, die mit französischer Hilfe erfolgen sollte. Unter dem Druck des neuen Versammlungsgesetzes von 1793 war es seither nicht mehr möglich gewesen, nach bewährtem Muster Versammlungen abzuhalten, auf denen die Ziele öffentlich diskutiert und dadurch in der Bevölkerung verbreitet werden konnten. Im Verlauf des Jahres 1795 vereinigten sich überdies United Irishmen und Defenders zu einer radikalrepublikanischen Bewegung in Irland, die auch über einen eigenen paramilitärischen Apparat verfügte.

Der Verbindungsmann der Organisation zum Direktorium in Paris war Theobald Wolfe Tone. Auch aus seinem nordamerikanischen Exil hatte er enge Kontakte nach Irland aufrechterhalten können und war schließlich aufgefordert worden, persönlich nach Frankreich zu reisen, um ,vor Ort' die Interessen der United Irishmen wahrzunehmen. Nachdem am 5. April 1795 französische und preußische Unterhändler in Basel Friedensbedingungen ausgehandelt hatten, war eine der großen Mächte aus der europäischen Anti-Revolutions-Koalition ausgeschieden. Aufgrund der verbesserten strategischen Lage Frankreichs konnte nun eine militärische Unterstützung der irischen Republikaner durchaus gewährt werden. Ohne die Aussicht auf auswärtige Militärhilfe wären alle Erhebungspläne der irischen Radikalen aufgrund der Kräfteverhältnisse im Lande von vornherein nur Makulatur gewesen. Es galt daher, diese Botschaft auf allen verfügbaren Kanälen dem Adressaten zu übermitteln. So reisten im Mai des Jahres zwei Mitglieder ihres Führungsrates nach Hamburg, um auch dort mit einem Agenten des Direktoriums die Bedingungen für eine französische Intervention in Irland zu erörtern. Zur selben Zeit war Tone bereits in Paris eingetroffen. Seine Eloquenz und sein Charisma öffneten ihm in kurzer Zeit die Türen zu einigen

19 Sean J. Connolly, Oxford Companion to Irish History, Oxford 2007, S. 434. 
maßgeblichen Angehörigen der französischen Regierung. Als besonders wichtig für den weiteren Verlauf der Planungen erwiesen sich bald seine Kontakte zum amtierenden Verteidigungsminister Lazare Carnot, der sich nach anfänglichem Zögern im Frühjahr 1796 den Argumenten des Iren anschloss. Dieser hatte stets den strategischen Nutzen betont, den Frankreich aus einer Landung in Irland ziehen könnte, zumal, laut Tone, $80 \%$ der irischen Bevölkerung, Katholiken und Presbyterianer, der Dubliner Regierung ablehnend gegenüberstünden. Zweifellos waren die Zahlen des irischen Gesandten zumindest geschönt, auch konnte er nicht garantieren, dass die irische Bevölkerung nach einer Landung französischer Truppen tatsächlichem ,mit fliegenden Fahnen' ins republikanische Lager wechseln würde, doch bot ein entschlossen durchgeführtes französisches Landungsunternehmen in Irland, das wenigstens nicht auf den offenen Widerstand der Bevölkerung treffen würde, in der Tat interessante militärstrategische Perspektiven. Schließlich hätte Großbritannien mit einem Abfall Irlands eine wichtige Nachschubbasis verloren, auch wäre von irischem Boden aus ein Angriff auf die relativ ungeschützte britische Flanke an der Irischen See leichter gewesen als im stark befestigten Südengland.

Dieser Erkenntnis konnten sich auch die übrigen Mitglieder des Direktoriums nicht widersetzen. Im Juni 1796 fiel die Entscheidung zugunsten der Entsendung eines Expeditionskorps nach Irland. ${ }^{20}$ Bald danach begann Carnot in Zusammenarbeit mit Lazare Hoche, einem der jungen und ehrgeizigen Generäle, die die Revolution hervorgebracht hatte, die konkreten Planungen und Vorbereitungen, die schnellstmöglich abgeschlossen sein sollten. Doch zögerte ein mangelhaftes Kommunikationssystem zwischen Paris und der Provinz die Aufstellung der Truppe sowie die Ausrüstung der notwendigen Schiffe über Gebühr hinaus, so dass das 15.000 Mann starke Korps erst im Dezember des Jahres einsatzbereit war. Anstatt besseres Wetter abzuwarten, entschloss sich Hoche zur sofortigen Durchführung der Operation. Schnell erwies sich dies als ein verhängnisvoller Fehler. Zwar gelang es der Royal Navy nicht, die französische Flotte nach dem Auslaufen aus Brest abzufangen, doch wurde diese von heftigen Winterstürmen auseinandergerissen. Lediglich ein Drittel der Schiffe gelangte bis in die Bantry Bay, nur um nach wenigen Tagen feststellen zu müssen, dass widrige Witterungsverhältnisse eine Landung der Truppen ausschlossen. Unverrichteter Dinge mussten die Schiffe schließlich in ihren Ausgangshafen zurückkehren.

Auch wenn diese Operation letztlich kläglich gescheitert war, zogen die britische Regierung und die Dubliner Administration energische Konsequenzen. Der Durchbruch der französischen Flotte hatte die Mängel des bisherigen Blockadesystems der kontinentalen Häfen offengelegt. Die Admiralität in London reagierte rasch

20 Zur geplanten Landung französischer Truppen in Irland vgl. Thomas Pakenham, The Year of Liberty. The Story of the Great Irish Rebellion of 1798, London 2000. 
und verstärkte die für die Blockade eingesetzten Schiffseinheiten sowie deren Taktik so nachhaltig, dass die französische Marine die Britischen Inseln bis zur endgültigen Niederlage Napoleons nicht mehr unmittelbar bedrohen konnte. Die enge Kommunikation zwischen den United Irishmen und dem Pariser Direktorium hatte ferner die Gefährlichkeit der irischen Radikalen für die bestehende Ordnung des Landes gezeigt. Eine Reihe von Notstandsverordnungen schränkte die erst vor kurzem gewährten Bürgerrechte wieder ein und erleichterte den Staatsorganen die Kontrolle verdächtiger Personen.

Die Aufstände des Jahres 1798, denen sich noch vereinzelte isolierte Revolten in den folgenden Monaten anschlossen, kosteten etwa 50.000 Menschen das Leben. Doch so blutig die Erhebungen auch waren und so viele nationalirisch inspirierte Mythen und Legenden später um diese Ereignisse gewebt werden mochten, die dadurch in den Rang eines einheitlichen und zentral gelenkten Aufstandes erhoben werden sollten, ändert das nichts an den historisch belegbaren Rahmenbedingungen. Es waren spontane Proteste, die zumeist von ehemaligen Anhängern der liberalen Politik Henry Grattans der späten 80er und frühen 90er Jahre des Jahrhunderts geführt wurden, denen die erreichten Reformen nicht genügten und deren radikale republikanische Ideale in der Auseinandersetzung mit einer zur Bewahrung des Status quo entschlossenen Staatsmacht chancenlos waren. Wesentlich größere Tragweite für den weiteren Verlauf der irischen Geschichte hatte denn auch eine politische Entscheidung des britischen Premierministers, an der dieser vermutlich schon seit einigen Jahren arbeitete, deren Realisierung indes letztlich erst vor dem Hintergrund des Sommers 1798 verständlich wird: dem Vollzug der Union. ${ }^{21}$ Er maß dem Verbleib Irlands im Empire, wie er seit seinem Amtsantritt im Jahre 1784 immer wieder betont hatte, eine erhebliche symbolische Bedeutung für den Bestand des britischen Weltreiches an sich bei. Besonders nach dem Abfall der amerikanischen Kolonien hätte die Unabhängigkeit Irlands einen Präzedenzfall für andere Teile des Reiches bieten können. Nachdem seine Initiative zur Schaffung eines einheitlichen anglo-irischen Wirtschaftsraumes bei gleichzeitiger Anerkennung bestimmter Formen nationaler Unabhängigkeit 1785 an den Parlamenten in London und Dublin gescheitert war, musste nun eine andere Lösung gefunden werden. Die politischen Entwicklungen und Verwicklungen nach Ausbruch der französischen Revolution hatten zwar zwischenzeitlich das irische Problem überlagert, angesichts einer drohenden, pointiert anti-britischen franko-irischen Republikanerallianz trat es nun umso deutlicher wieder hervor.

Doch auch wenn vor dem Hintergrund der zurückliegenden Ereignisse die Notwendigkeit einer engen anglo-irischen Kooperation in Sicherheitsfragen von den meisten Angehörigen des Dubliner Parlaments nicht bestritten wurde, wenn diese

$21 \mathrm{Zu}$ Pitts Unionsplänen und deren Durchführung vgl. z. B. Michael J. Turner, Pitt the Younger. A Life, London 2005. 
die Kooperation darüber hinaus auch aus ökonomischen Erwägungen gar nicht in Frage stellen wollten, stand die weitaus größte Mehrheit des irischen Parlaments dem Vollzug einer Union, also der Auflösung einer eigenständigen irischen Legislative, ablehnend gegenüber. Sicher: Pitt besaß einige Verbündete jenseits der Irischen See, besonders unter den konservativen Protestanten, die sich von der Union einen verbesserten Schutz protestantischer Privilegien erhofften, schließlich hätten sich die Protestanten dann nicht mehr in einer Minderheitsposition befunden, sondern wären, wie die Mehrheit der Bevölkerung, Angehörige der Staatskirche gewesen. Auch sprachen viele ökonomische Argumente für die Vereinigung der beiden Königreiche, wäre es unter einem einheitlichen staatlichen Dach doch beispielsweise für viele potentielle britische Investoren leichter gewesen, ihr Geld auch in Irland anzulegen, was fraglos zu einer Verbesserung des irischen Lebensstandards beigetragen hätte. Doch war dies im Dubliner Parlament noch die Meinung einer (wenn auch starken) Minderheit, die Mehrheit, das stand spätestens im Februar 1799 fest, als das irische Parlament erstmals das Für und wider einer Union mit Großbritannien erörterte, teilte diese Meinung nicht, denn der Entwurf wurde mit 109 zu 104 Stimmen abgelehnt. Charles Cornwallis, seit 1798 Vizekönig in Irland, erhielt daher vom Premierminister den Auftrag, nachhaltige Überzeugungsarbeit zu leisten und, wenn diese keinen Erfolg haben sollte, nötigenfalls auch mit großzügigen Geldmitteln und der Aussicht auf weitere persönliche Vorteile um Unterstützung für die Unionspläne zu werben.

Die vizekönigliche Behörde musste in den folgenden Wochen auf vielen Ebenen um Sympathien für die Pläne Pitts werben, die zwischenzeitlich auch die Zustimmung des britischen Parlaments gefunden hatten. Erste Zielgruppe waren natürlich die Abgeordneten des irischen Parlaments, ohne deren Zustimmung eine Union nicht möglich gewesen wäre. Darüber hinaus galt es, sich möglichst großer Unterstützung durch die Bevölkerung zu versichern, von der man sich nachhaltige Rückwirkung auf die Haltung der Parlamentarier erhoffte. Dies jedoch wussten auch die entschiedenen Unionsgegner, so dass beide Gruppen mit beachtlichem Aufwand die Bürger des Landes über die Vor- und Nachteile eines Vereinigten Königreiches von Großbritannien und Irland informierten. In großem Umfang nutzten sie dabei die vorhandenen Publikationsorgane sowie Flugblätter und Plakate. Daneben boten sie allen Interessierten die Gelegenheit, sich auf Versammlungen und anderen öffentlichen Veranstaltungen zu informieren. Die Wirkung dieser Kampagnen war zweifellos groß, dennoch sind auch nur annähernd zuverlässige Schätzungen ihres tatsächlichen Wirkungsgrades nicht möglich, dazu waren die Einflüsse regionaler, sozialer oder ökonomischer Partikularinteressen zu unterschiedlich. Lediglich einige allgemeine Stimmungstrends sind anhand von zeitgenössischen Stimmungsberichten und anderen Aufzeichnungen nachvollziehbar. ${ }^{22}$

22 Zur Abstimmung über die Union vgl.: Elvert, 2003 (wie Anm. 15), Kap.5. 
So scheinen sich die Bürger Dublins, um die herausragende Rolle der Stadt als Metropole eines eigenen Königreiches besorgt, mehrheitlich gegen eine Union ausgesprochen zu haben, während die Stimmung in Cork und Belfast in dieser Frage eher geteilt war. Hier beeinflussten offensichtlich Überlegungen über die Vor- und Nachteile einer engeren ökonomischen Kooperation das Stimmungsbild. Die Protestanten waren in der Unionsfrage tief gespalten. Während viele Anhänger der Staatskirche die Sicherheit zu schätzen wussten, die ihnen im Rahmen eines Vereinten Königreiches geboten werden konnte, fürchteten die Presbyterianer mehrheitlich den Verlust von Handlungsfreiheiten, die sich im Laufe der Zeit in ihren Hauptsiedlungsgebieten, vorwiegend im irischen Norden, erstritten hatten. Auf den ersten Blick erstaunlich sind die überlieferten katholischen Reaktionen. Einmal abgesehen von den wenigen Radikalrepublikanern, die die Aufstände des Sommers 1798 und die anschließenden Verfolgungen durch die Staatsmacht überlebt hatten, und ferner abgesehen von einigen engagierten Anhängern des liberal-nationalen Kurses Henry Grattans waren die Ansichten in der Unionsfrage durchaus differenziert. Offenbar stießen die Hinweise des Vizekönigs auf die Gewährung völliger Gleichberechtigung nach Verabschiedung des Unionsgesetzes gerade bei vielen kirchlichen Würdenträgern auf großes Interesse. Bei ihnen waren die Erinnerungen an die Ausschreitungen gegen katholische Priester im revolutionären Frankreich noch in sehr guter Erinnerung, die Tausenden das Leben gekostet hatten. Angesichts der immer noch virulenten Bedrohung der Britischen Inseln durch Frankreich schien die relative Sicherheit im Rahmen eines gestärkten Großbritannien mit möglicher Aussicht auf Gleichberechtigung eine bessere Perspektive zu bieten als die relative Unsicherheit in einem revolutionär-republikanischen System.

Andere Mittel mussten zur Umstimmung der Abgeordneten angewendet werden. Da der Entwurf der Unionsakte im Unterhaus 100 (von 658), im Oberhaus 32 Sitze für die Repräsentanten Irlands vorsah, bedeutete dies für drei von vier Mitgliedern der beiden irischen Häuser zunächst den Abschied von der Tätigkeit als Parlamentarier. Auch wenn sich nur wenige der Betroffenen von solchen Überlegungen leiten ließen, erlaubten die knappen Mehrheitsverhältnisse kein Risiko. Der Vizekönig und seine Bevollmächtigten prüften jeden Einzelfall, garantierten stets die soziale Absicherung und in besonders hartnäckigen Fällen sogar noch einen Profit für den Fall, dass die Unionsakte angenommen werden würde. Es gab also bei dem Verfahren um das anglo-irische Unionsgesetz einige Fälle von Bestechung. Allerdings muss rückblickend festgestellt werden, dass sich die meisten Abgeordneten des Dubliner Parlaments bei ihrer Entscheidung für oder gegen das Vereinigte Königreich von Großbritannien und Irland letztlich doch in erster Linie von ihrer Überzeugung leiten ließen, so dass die Abstimmungsergebnisse der zweiten (118:73) und dritten Lesung (158 : 87) in etwa die tatsächlichen Meinungsverhältnisse im Parlament repräsentierten. Dafür spricht auch das Tempo, mit dem man in Irland nach Inkrafttreten der 
Unionsakte am 1. Januar 1801 wieder zur politischen Tagesordnung überging und mit dem sich die neuen Abgeordneten in Westminster in ihren Rollen zurechtfanden. Offensichtlich hatte die irische Bevölkerung in ihrer großen Mehrheit kein Interesse mehr an irgendwelchen radikalrepublikanischen Umsturzplänen, sondern erhoffte sich nach langen Jahren politischer und ökonomischer Instabilität von der Union mit Großbritannien nunmehr inneren und äußeren Frieden, Freiheit und Wohlstand. Zum Zeitpunkt des Vollzugs der Union konnte allerdings niemand ahnen, dass es bis dahin noch ein sehr weiter Weg sein würde. 